\title{
О ЗАКОНЕ РАСПРЕДЕЛЕНИЯ МЕСТОРОЖДЕНИЙ УГЛЕВОДОРОДОВ ПО МАССЕ
}

\author{
Лившиц Валерий Рафаилович, \\ livshic.vr@mail.ru
}

Институт нефтегазовой геологии и геофизики им. А.А. Трофимука СО РАН, Россия, 630090, г. Новосибирск, пр. Академика Коптюга, 3.

\begin{abstract}
Актуальность исследования обусловлена той, исключительно важной ролью, которую играет вероятностное распределение скоплений углеводородов по массе (усеченное распределение Парето) при количественном прогнозе нефтегазоносности в слабоизученных и зрелых нефтегазоносных бассейнах. Знание этого распределения позволяет осуществлять прогноз количества и суммарных ресурсов углеводородов в любых заданных интервалах крупности, прогноз величины запасов невыявленных скоплений и последовательности их открытий.

Цель: для закона распределения залежей углеводородов по массе - усеченного распределения Парето, получить соответствующее вероятностное распределение для месторождений по массе уелеводородов.

Методы: аналитические методы теории вероятностей, метод статистических испытаний, статистическая обработка эмпирических данных.

объект: нефтегазоносные бассейны, запасы нефти и газа залежей, месторождений, количество залежей в месторождениях законы распределения скоплений углеводородов по массе.

Результаты. Показано, что степенное распределение вероятностей (классическое распределение Парето, усеченное распределение Парето) не может играть роль распределения скоплений углеводородов по массе одновременно и для залежей, и для месторождений. Применение метода статистических испытаний позволило получить условные вероятностные распределения месторождений уелеводородов по массе при фриксированном числе залежей в них. На основе обработки эмпирических данных по четырем крупнейшим нефртегазоносным провинциям России (Волго-Уральской, Западно-Сибирской, ТиманоПечорской и Северо-Кавказской) были найдены оценки распределений месторождений углеводородов по количеству залежей в них. Исходя из закона распределения залежей углеводородов по их крупности - усеченного распределения Парето, найден закон распределения углеводородов по массе для месторождений. Показано, что полученное вероятностное распределение, не являясь, строго говоря, степенным, оказывается весьма близким к нему.
\end{abstract}

\section{Ключевые слова:}

Нефттегазоносная провинция, скопления, залежи, месторождения углеводородов, вероятностное распределение месторождений и залежей углеводородов по массе, классическое распределение Парето, усеченное распределение Парето.

\section{Введение}

Современные представления о преобразовании углеводородистого вещества в земной коре $[1,2]$ позволяют утверждать, что процессы генерации, миграции, аккумуляции и рассеяния углеводородов (УВ) носят стохастический характер. Как следствие, стохастическим оказывается и результат процесса формирования скоплений УВ в ловушках, так, что масса скопления представляет собой случайную величину, математическое описание которой дается ее вероятностным распределением.

Закон распределения скоплений УВ по массе играет исключительно важную роль как для теории нафтидогенеза, так и для практики количественной оценки перспектив нефтегазоносности. В теоретическом плане этот закон должен логически входить в общую теорию нафтидогенеза $[1,2]$, а в практическом плане такой закон позволяет оценивать количество и суммарные ресурсы УВ в любых заданных интервалах крупности, осуществлять прогноз вероятных запасов неоткрытых месторождений и последовательность их выявления [3-5].

Первые попытки эмпирически установить закон распределения скоплений УВ по массе на основе обработки величин запасов открытых месторождений в хорошо изученных нефтегазоносных бассейнах (НГБ) привел к ошибочному заключению о логарифмически нормальном законе их распределения [6]. Ошибочность этого результата заключается в том, что в силу действия геологоразведочного фильтра [7] совокупность величин запасов открытых скоплений не является репрезентативной выборкой для величин запасов всех скоплений бассейна.

Последующая статистическая обработка данных по Волго-Уральскому НГБ с учетом действия геологоразведочного фильтра позволил В.И. Шпильману установить степенной характер распределения месторождений по запасам, названный им законом «обратных квадратов» [6].

Дальнейшие исследования по множеству хорошо изученных НГБ мира, выполненные А.Э. Конторовичем и В.И. Деминым, привели их к более общему выражению для распределения месторождений нефти и газа по величине запасов - усеченному распределению Парето $[4,5]$. Распределение Парето, усеченное на отрезке $\left[x_{0}, x_{m}\right]$, имеет вид

$$
\varphi(x)=C\left(\frac{1}{x^{\lambda}}-\frac{1}{x_{m}^{\lambda}}\right),
$$

где $1<\lambda<3$ - параметр распределения, $C=\frac{(\lambda-1) x_{m}^{\lambda}}{x_{0}\left[\lambda-1+\left(\frac{x_{m}}{x_{0}}\right)^{\lambda}\right]-\lambda x_{m}}-$ нормирующий множитель.

Наконец, более поздние исследования Н.А. Крылова, А.Г. Алексина, Ю.Н. Батурина [8], а также Ю.А. Арсирия, 
Б.П. Кобышева, Д.И. Чупрынина [9] подтвердили справедливость результатов В.И. Шпильмана, А.Э. Конторовича, В.И. Демина. К аналогичному заключению о характере распределения скоплений УВ по величине запасов пришли и зарубежные исследователи [10-14].

Поскольку степенной характер этого распределения оказывается справедливым независимо от геологического строения и истории развития НГБ, естественно предположить, что этот закон носит универсальный, фундаментальный характер $[1,2]$.

В настоящее время степенной характер распределения скоплений УВ по массе не вызывает возражений у подавляющего большинства исследователей, однако остается открытым вопрос о генезисе этого распределения, а также о том, распределение каких именно скоплений описывает этот закон: залежей или месторождений, поскольку статистическая обработка величин запасов открытых скоплений может относиться как к залежам, так и к месторождениям УВ. В результате возникла ситуация, когда неявно предполагается справедливость степенного закона как для залежей, так и для месторождений УВ. Однако распределение Парето не может одновременно играть роль вероятностного распределения по массе УВ и для залежей, и для месторождений.

Действительно, залежь, определяемая как единичное скопление нефти и газа, заполняющее ловушку полностью или частично, представляет собой единый физический объект, в котором протекают процессы аккумуляции и рассеяния нефти. Для таких объектов был разработан целый ряд схем, приводящих к степенному характеру распределения залежей УВ по массе В основе этих схем лежат процессы аккумуляциидиссипации УВ в ловушках [15], процессы латеральной миграции УВ $[16,17]$, процессы в неравновесных динамических системах [18] и, наконец, несомненная связь степенного распределения с фрактальными объектами [19]. Какая бы из этих схем не реализовывалась в действительности, в любом случае, с большой долей уверенности можно утверждать, что распределение УВ в залежах подчиняется степенному распределению.

Месторождение же (если оно не однозалежное) группа залежей, имеющих в проекции на земную поверхность полное или частичное перекрытие своих контуров нефтегазоносности или же группа залежей, разобщенных в плане, но контролируемых одной локальной структурой. Таким образом, месторождение является объектом не столько физическим, сколько геометрическим, объединяющим несколько залежей по принципу их геометрического расположения, тогда как характер процессов аккумуляции и рассеяния в различных залежах одного месторождения может быть существенно различным. При этом масса месторождения есть просто сумма масс УВ залежей, составляющих это месторождение.

Закон распределения суммы случайных величин может совпадать (с точностью до параметров сдвига и масштаба) с законом распределения слагаемых только в том случае, если эти законы являются устойчивыми [20]. Сами устойчивые распределения относятся к классу так называемых безгранично делимых распре- делений, а такие распределения не могут быть сосредоточены на конечном интервале [20]. Из этого следует, что усеченное распределение Парето, не являясь безгранично делимым, не является и устойчивым. Таким образом, если принять, что распределение залежей нефти и газа по массе подчиняется усеченному распределению Парето, то распределение месторождений не может подчиняться этому распределению.

Заметим также, что классическое распределение Парето является безгранично делимым [20] и поэтому может играть роль вероятностного распределения для суммы случайных величин, т. е. выступать в качестве распределения для массы месторождений УВ, но в этом случае оно не будет распределением залежей, поскольку не является устойчивым распределением.

Найдем распределение месторождений УВ по массе в предположении, что распределение залежей в НГБ подчиняется усеченному распределению Парето. Очевидно, что задача сводится к нахождению распределения суммы случайных величин с известной плотностью.

Пусть $Z$ - масса УВ месторождения, т. е. сумма масс залежей. Очевидно, что распределение величины $Z$ зависит от количества залежей в месторождении, т. е. описывается условной плотностью распределения месторождений с $k$ залежами $f(z \mid k)$.

Тогда безусловная плотность распределения месторождений по величине запасов в НГБ может быть найдена по формуле полной вероятности

$$
f(z)=\sum_{k=1}^{m} P_{k} f(z \mid k),
$$

где $P_{k}$ - вероятность месторождения с $k$ залежами, $m$ - максимально возможное количество залежей в месторождении.

Условные плотности распределения месторождений УВ по массе с фиксированным количеством залежей

Очевидно, что условная плотность распределения месторождений с одной залежью совпадает с плотностью распределения залежей $\varphi(x)$ так, что $f(z \mid 1)=\varphi(z)$.

Найдем плотность распределения по массе месторождений, состоящих из двух залежей $f(z \mid 2)$, при условии, что распределение этих залежей подчиняется усеченному распределению Парето $\varphi(x)$. Запасы такого месторождения $Z$ равны сумме двух случайных величин - масс УВ залежей, распределенных как $\varphi(x)$, а их плотность распределения (в предположении независимости масс залежей) выражается интегралом свертки [20]

$$
f(z \mid 2)=\int_{0}^{z} \varphi(x) \varphi(z-x) d x .
$$

Для случая, когда функция $\varphi(x)$ задана на положительном отрезке $x_{0} \leq x \leq x_{m}$, свертка примет вид

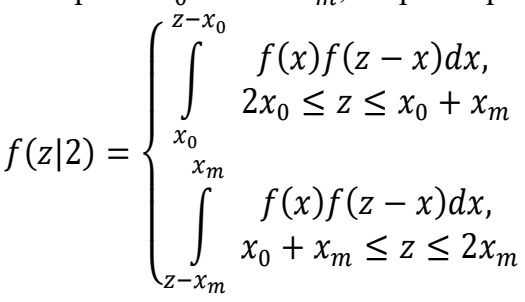


Подставляя в это выражение плотность усеченного распределения Парето $\varphi(x)$, получим

$$
\left\{\begin{array}{c}
f(z \mid 2)= \\
C^{2}\left[\begin{array}{c}
\frac{d x}{x^{\lambda}(z-x)^{\lambda}}+ \\
\int_{x_{0}}^{z-x_{0}}+\frac{2}{x_{m}^{\lambda}(\lambda-1)}\left(\frac{1}{\left(z-x_{0}\right)^{\lambda-1}}-\frac{1}{x_{0} \lambda-1}\right)+ \\
+\frac{z-2 x_{0}}{x_{m}^{2 \lambda}}
\end{array}\right], \\
C^{2}\left[\begin{array}{c}
\int_{z-x_{m}}+\frac{d x}{x_{m}^{\lambda}(\lambda-1)}\left(\frac{1}{x_{m}^{\lambda-1}}-\frac{1}{\left(z-x_{m}\right)^{\lambda-1}}\right) \\
+\frac{2 x_{m}-z}{x_{m}^{2 \lambda}}
\end{array}\right] . \\
\text { при } x_{0}+x_{m} \leq z \leq 2 x_{m}
\end{array}\right] .
$$

Здесь первое слагаемое соответствует случаю классического Парето, для перехода к которому следует положить $x_{m} \rightarrow \infty$. Поскольку правая граница усеченного распределения Парето, как правило, величина достаточно большая, то разница между усеченным и классическим Парето оказывается весьма незначительной. При больших $z$ распределение имеет степенную асимптотику, так что его правый «хвост» ведет себя так же, как и распределение Парето.

Интеграл $\int \frac{d x}{x^{\lambda}(z-x)^{\lambda}}$ в общем случае не выражается через элементарные функции. Однако при $\lambda=2$ подинтегральная функция оказывается рациональной и интеграл может быть записан в конечном виде

$$
\begin{gathered}
\int \frac{d x}{x^{2}(z-x)^{2}}=\frac{1}{z^{2}(z-x)}- \\
-\frac{1}{z^{2} x}+\frac{2}{z^{3}} \ln \frac{x}{z-x}
\end{gathered}
$$

так что для случая $\lambda=2$ плотность распределения месторождения с двумя залежами будет иметь вид

$$
f(z \mid 2)=\left\{\begin{array}{c}
C^{2}\left[\begin{array}{c}
\frac{2}{z^{2}}\left(\frac{1}{x_{0}}-\frac{1}{z-x_{0}}+\frac{2}{z} \ln \frac{z-x_{0}}{x_{0}}\right)+ \\
+\frac{2}{x_{m}^{2}}\left(\frac{1}{z-x_{0}}-\frac{1}{x_{0}}\right)+\frac{z-2 x_{0}}{x_{m}^{4}}
\end{array}\right], \\
C^{2}\left[\begin{array}{c}
\frac{2}{z^{2}}\left(\frac{1}{x_{m}}-\frac{1}{z-x_{m}}+\frac{2}{z} \ln \frac{x_{m}}{z-x_{m}}\right)+ \\
+\frac{2}{x_{m}^{2}}\left(\frac{1}{x_{m}}-\frac{1}{z-x_{m}}\right)+\frac{2 x_{m}-z}{x_{m}^{4}} \\
x_{0}+x_{m} \leq z \leq 2 x_{m}
\end{array}\right] .
\end{array}\right.
$$

При $\lambda \neq 2$ значение этого интеграла может быть найдено численным интегрированием.

Для нахождения условных плотностей распределения по запасам месторождений с числом залежей больше двух $f(z \mid k), k>2$ можно воспользоваться методом Монте-Карло, генерируя $k$ случайных чисел, распределенных в соответствии с усеченным распределением Парето и имитирующих массы залежей. Сумма этих чисел будет имитировать массу место- рождения, составленного из $k$ залежей. Метод генерации таких чисел подробно рассмотрен в [4]. Многократное повторение этой процедуры позволяет получить статистическое распределение месторождений по массе с заданным количеством залежей.

На рис. 1 приведены условные плотности распределения вероятности массы месторождения при различных значениях числа залежей в нем $k$ и параметра $\lambda$ усеченного распределения Парето. Как видно из рисунка, плотности распределения месторождений имеют моду и это наиболее вероятное значение массы увеличивается с ростом числа залежей.

Если бы в НГБ отсутствовали однозалежные месторождения, распределение месторождений УВ по величине запасов в таком бассейне имело бы моду. Однако, как показано ниже, большая часть месторождений бассейна являются именно однозалежными.

\section{Безусловное распределение месторождений УВ} по массе в НГБ

Как видно из формулы полной вероятности (*), для нахождения безусловного распределения месторождений УВ по массе необходимо знание априорных вероятностей $P_{k}, k=1, \cdots, m$. Для получения статистических оценок этих величин можно воспользоваться информацией по хорошо изученным нефтегазоносным провинциям. На рис. 2 приведены оценки этих вероятностей для Волго-Уральской, ЗападноСибирской, Тимано-Печорской и Северо-Кавказской провинций, для $m=10$ (месторождения с количеством залежей до 10 составляют более 80 \% от общего числа месторождений провинции, для месторождений с $m>10$, в силу малого их количества, выборочные флуктуации не позволяют получить оценку приемлемой точности). Интересно отметить, что все четыре зависимости практически совпадают и хорошо аппроксимируются степенной кривой.

На рис. 3 показаны безусловные плотности распределения месторождений для различных значений параметра $\lambda$ усеченного распределения Парето, в соответствии с которым распределены массы залежей, и при оценках априорных вероятностей, полученных для Волго-Уральской провинции.

Как видно из рисунка, полученные плотности представляют собой амодальные, асимметричные распределения, которые хорошо аппроксимируются степенной зависимостью. Тем не менее эти плотности не являются степенным распределением, и их проверка по статистическим критериям с очень высокой степенью достоверности отклоняет эту гипотезу.

Внешнее сходство со степенным распределением обусловлено тем, что влияние на сумму (*) слагаемых c $k>1$ оказывается слабым по сравнению со слагаемым $k=1$. Это связано, во-первых, со значительной долей однозалежных месторождений, которые распределены в соответствии с усеченным распределением Парето, и, во-вторых, с тем, что модальные значения условных плотностей наиболее значительны для малых $k$, т. е. приходятся на те значения массы, для которых плотность распределения однозалежных 
месторождений велика. Сказанное иллюстрируется на рис. 4, где для $\lambda=2$ показаны произведения услов- ных плотностей на соответствующие веса $P_{k}$ для $k=1,2,3,4$ и их сумма.
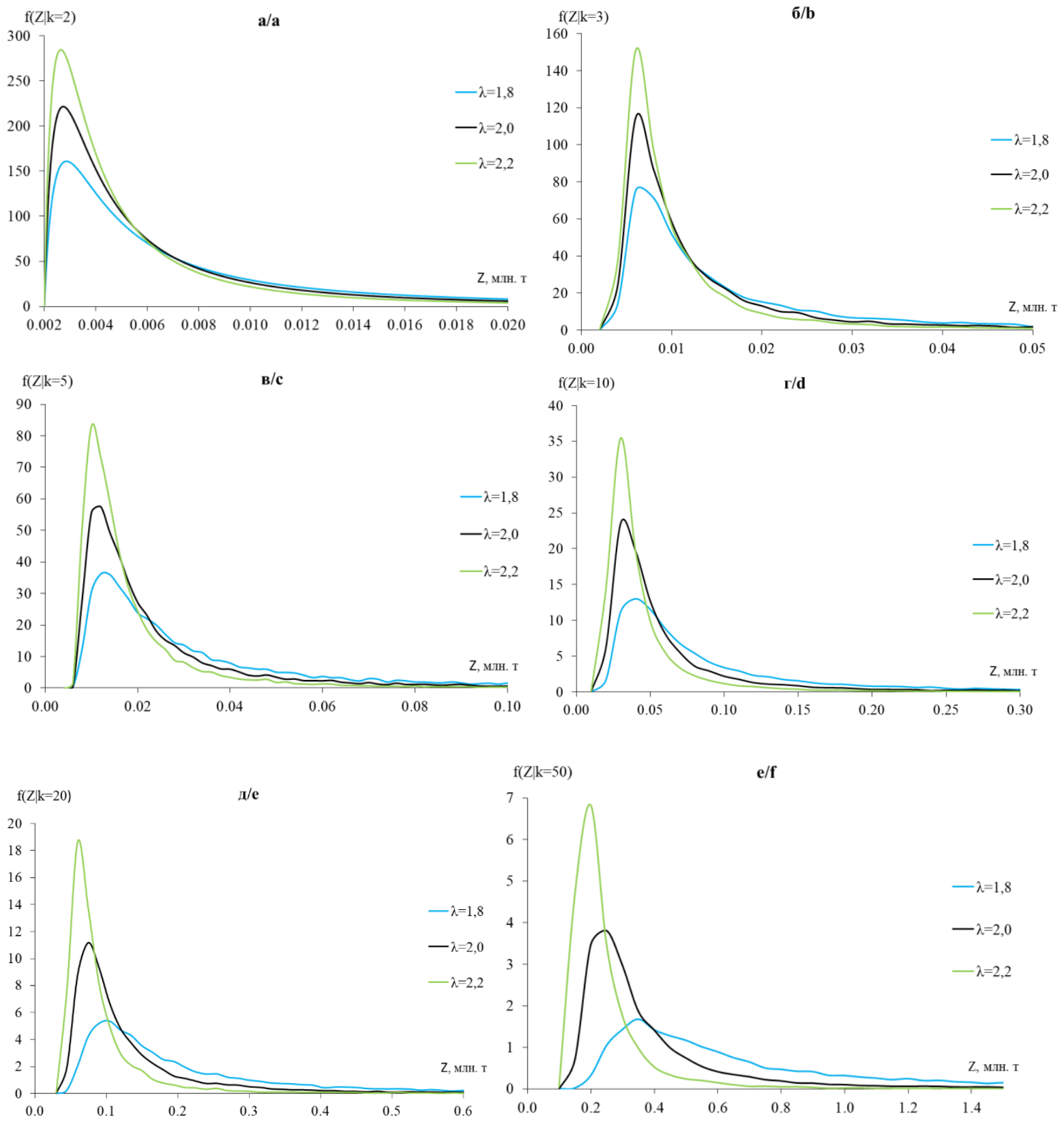

Рис. 1. Условная плотность распределения месторождений УВ по массе при фиксированном числе залежей $k$ и различном $\lambda$ : a) $k=2$, б) $k=3$, в) $k=5$, г) $k=10$, д) $k=20$, е) $k=50$

Fig. 1. Conditional density of size distribution of oil and gas fields for a fixed number of pools $k$ and different $\lambda$ : a) $k=2$, b) $k=3$, c) $k=5, d$ ) $k=10$, e) $k=20, f) k=50$

\section{Заключение}

Выполненное исследование позволило сделать следующие заключения:

1. Хотя распределение залежей нефти и газа по величине запасов в нефтегазоносном бассейне подчиняется усеченному распределению Парето, это распределение не может быть распределением по массе для месторождений бассейна.

2. Распределение числа месторождений по количеству залежей в них практически одинаково для четырех крупнейших нефтегазоносных провинций России и хорошо аппроксимируется степенным распределением.

3. Распределение месторождений углеводородов в нефтегазоносном бассейне по величине их запасов, не являясь, строго говоря, степенным распределением, оказывается весьма близким к нему, причем основное отличие лежит в области мелких и мельчайших скоплений. 


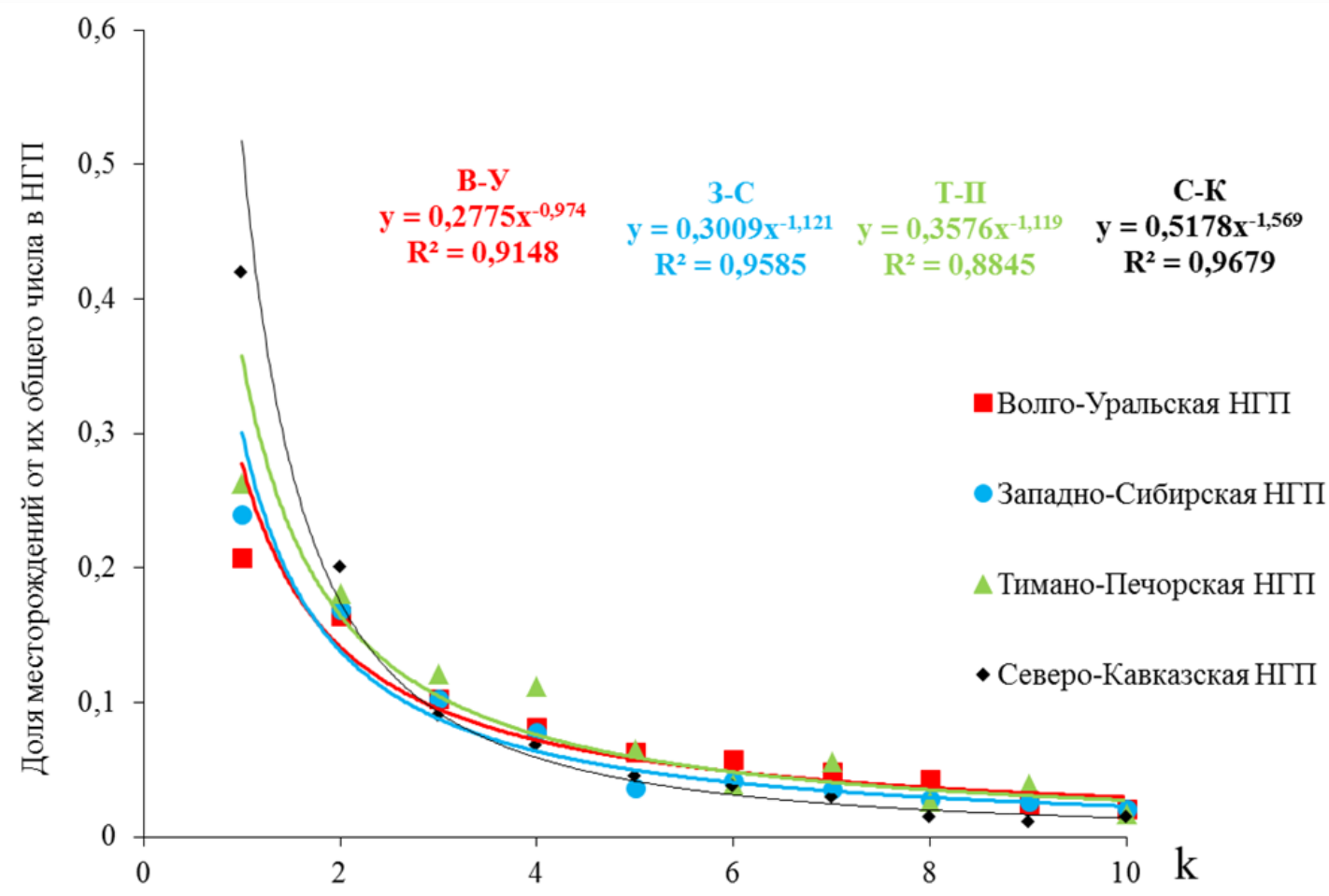

Рис. 2. Зависимость доли месторождений (от их общего числа) для Волго-Уральской, Западно-Сибирской, ТиманоПечорской и Северо-Кавказской НГП от количества залежей в месторождении

Fig. 2. Proportion of oil and gas fields (of their total number) for the Volga-Ural, West Siberian, Timan-Pechora and NorthCaucasian petroleum provinces as a function of the number of pools in each field

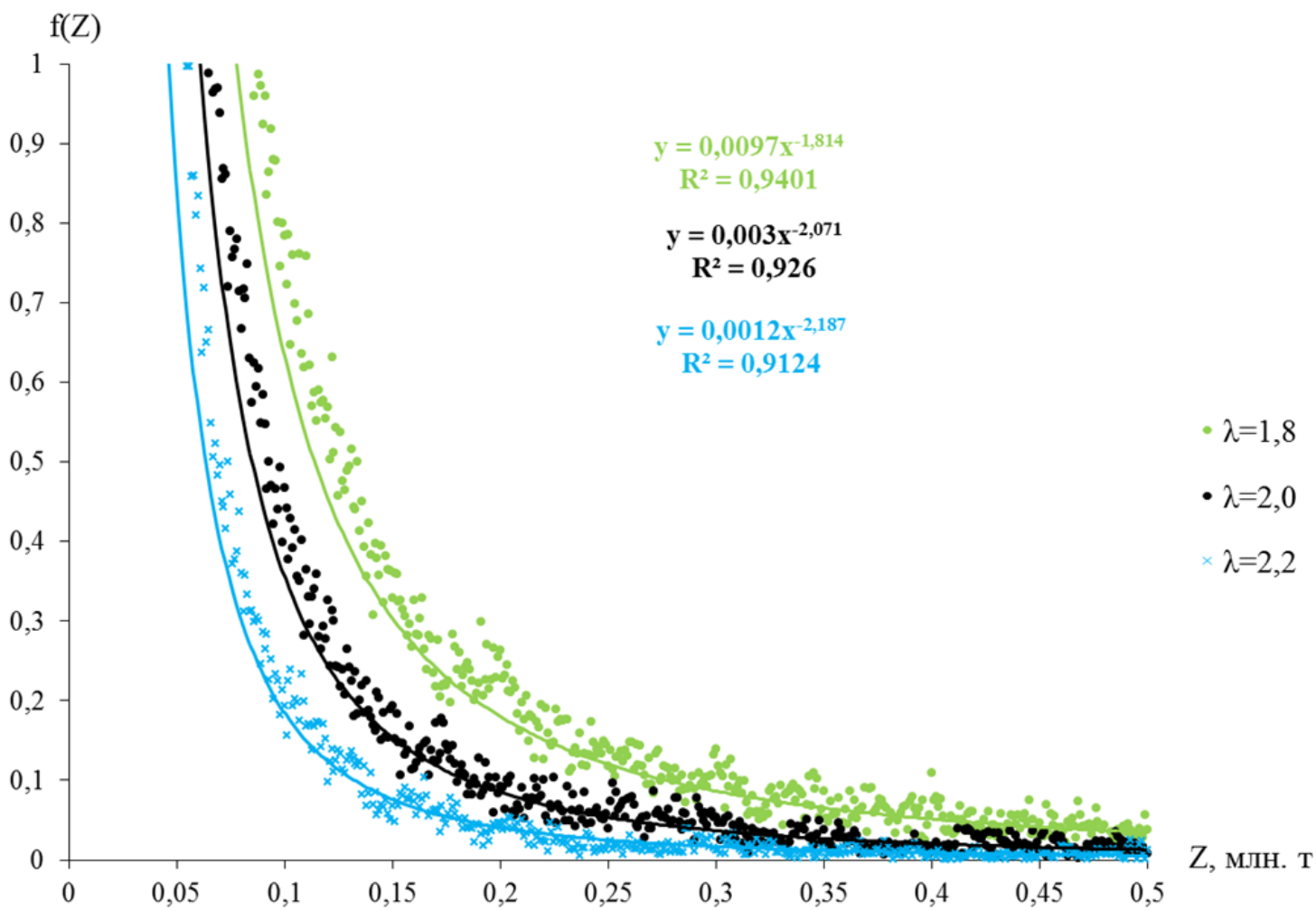

Рис. 3. Безусловные плотности распределения месторождений по массе для различных значений параметра $\lambda$ Fig. 3. Unconditional densities of the distribution of sizes of oil and gas fields for different values of the parameter $\lambda$ 


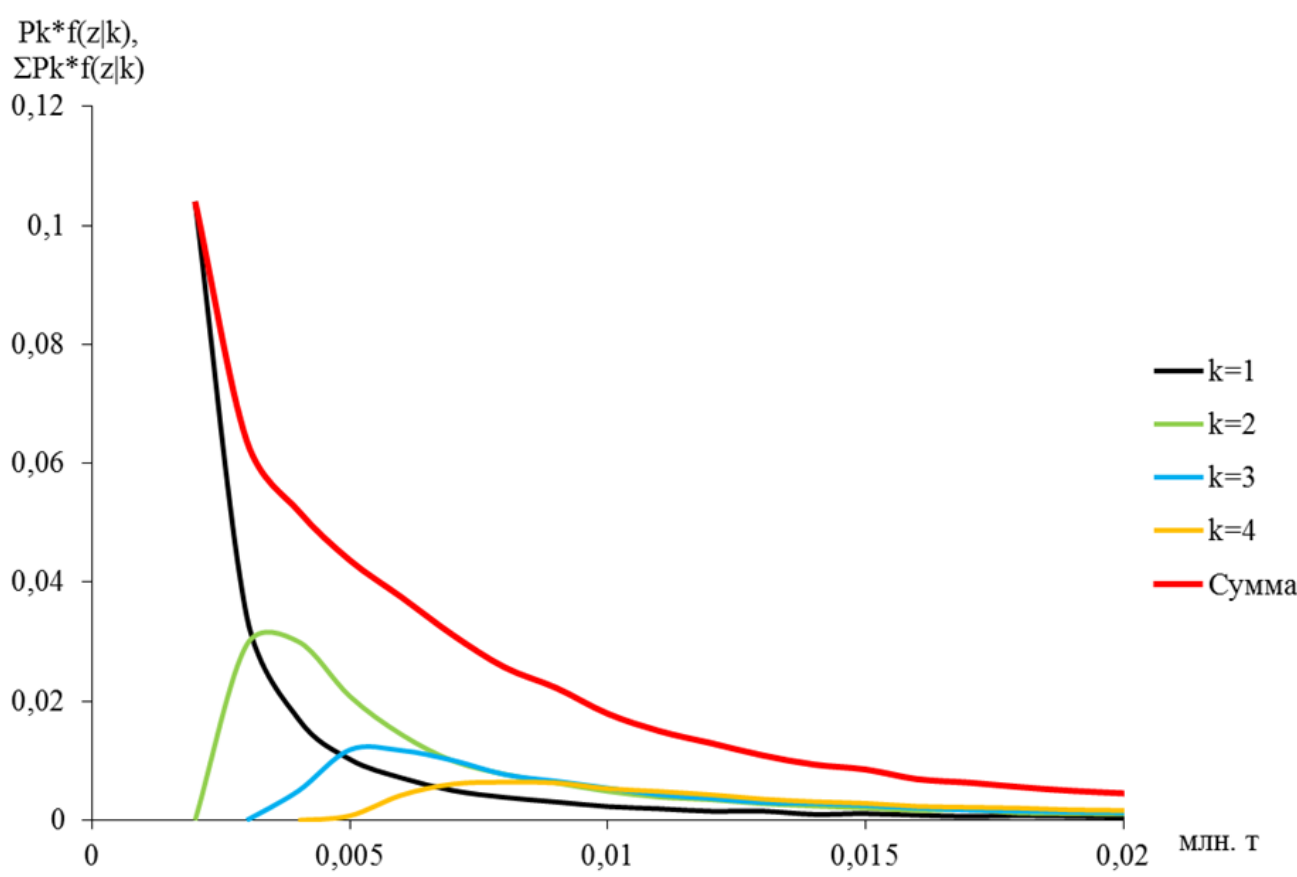

Рис. 4. Иллюстрация возникновения амодального характера распределения месторождений УВ по запасам

Fig. 4. Illustration of the amodal nature of distribution of oil and gas fields by their reserves sizes

\section{СПИСОК ЛИТЕРАТУРЫ}

1. Kontorovich A.Eh. Formation of oil and gas in the Earth's crust// Petroleum geology handbook / Ed. by N.A. Eremenko. - Los Angeles, California, U.S.A.: OSI Publications, 1991. - P. 548-569.

2. Конторович А.Э. Осадочно-миграционная теория нафтидогенеза: состояние на рубеже XX и XXI вв., пути дальнейшего развития // Геология нефти и газа. - 1998. - № 10. - С. 8-16.

3. Конторович А.Э., Лившиц В.Р. Новые методы оценки, особенности структуры и пути освоения прогнозных ресурсов нефти зрелых нефтегазоносных провинций (на примере Волго-Уральской провинции) // Геология и геофизика. - 2017. № 12. - C. $1835-1852$.

4. Kontorovich A., Domain V., Livshitc V. Size distribution and dynamics of oil and gas field discoveries in petroleum basins // American Association of Petroleum Geologists Bulletin. - 2001. V. 85 (9). - P. 1609-1622.

5. Конторович А.Э., Демин В.И. Прогноз количества и распределения по запасам месторождений нефти и газа // Геология и геофизика. - 1979. - № 3. - С. 26-46.

6. Kaufman G.M., Balcer Y., Kruit D.A. Probabilistic model of oil and gas discovery // American Association of Petroleum Geologists. - 1975. - № 1. - P. 113-142.

7. Шпильман В.И. Количественный прогноз нефтегазоносности. - М.: Недра, 1982. -215 с

8. Крылов Н.А., Алексин А.Г., Батурин Ю.Н. Задачи и пути ускорения научно-технического прогресса при поисках нефти в районах с высокой разведанностью недр // Геология нефти и газа. - 1986. - № 7. - С. 1-7.

9. Прогноз размеров и числа неоткрытых залежей УВ и методика их поисков в ДДВ / Ю.А. Арсирий, Б.П. Кабышев, Д.И. Чупрынин, А.Ф. Шевченко, З.П. Шевякова // Геология нефти и газа. - 1986. - № 10. - С. 42-46.

10. Schuenemeyer J.H., Lawrence J., Drew. A. Procedure to estimate the parent population of the size of oil and gas fields as revealed by a study of economic truncation // Mathematical Geology. 1983. - V. 15 (1). - P. 145-160.

11. Houghton J.C. Use of the truncated shifted Pareto distribution in assessing size distribution of oil and gas fields // Mathematical Geology. - 1988. - № 8. - P. 907-937.

12. Merriam D.F., Drew L.J., Schuenemeyer J.H. Zipf's law: a viable geological paradigm? // Natural Resources Research. - 2004. № 13. - P. 265-271.

13. Laherrere J. Distribution of field sizes in a petroleum system: parabolic fractal, lognormal or stretched exponential? // Marine and Petroleum Geology. - 2000. - V. 17 (4). - P. 539-546.

14. Smith J.L., Ward G.L. Maximum likelihood estimates of the size distribution of North Sea Oil Fields // Mathematical Geology. 1981. - V. 13 (5). - P. 399-413.

15. Бурштейн Л.М. Возможный механизм формирования распределения скоплений углеводородов по крупности // Геология и геофизика. - 2004. - Т. 45. - № 7. - С. 815-825.

16. Лившиц В.Р. Латеральная миграция углеводородов как возможный механизм формирования степенного распределения их скоплений по массе // Геология и геофизика. - 2017. - № 3-4. C. $372-383$.

17. Лившиц В. Р. Имитационная стохастическая модель латеральной миграции углеводородов // Геология и геофизика. 2014. - Т. 55. - № 5-6. - С. 906-917.

18. Родкин М.В., Рундквист Д.В. Геофлюидодинамика. Приложение к сейсмологии, тектонике, процессам рудо и нефтегенеза. - Долгопрудный: ИД «Интеллект», 2017. - 282 с.

19. Деменок С.Л. Фрактал: между мифом и ремеслом. - СПб.: ООО «Страта», 2018. - 87 c.

20. Феллер В. Введение в теорию вероятностей и ее приложения. - М.: Мир, 1984. - Т. 2. - 751 с.

Поступила 22.02.2020 2.

\section{Информация об авторах}

Ливииц В.P., доктор геолого-минералогических наук, главный научный сотрудник Института нефтегазовой геологии и геофизики им. А. А. Трофимука СО РАН. 
UDC 553.98

\title{
LAW OF SIZE DISTRIBUTION OF OIL AND GAS FIELDS
}

\author{
Valery R. Livshits, \\ livshic.vr@mail.ru \\ Trofimuk Institute of Petroleum Geology and Geophysics, Siberian Branch Russian Academy of Sciences, \\ 3, Akademician Koptyug avenue, Novosibirsk, 630090, Russia.
}

Relevance of the study is related to the extremely important role played by the probabilistic distribution of the sizes of oil and gas fields (truncated Pareto distribution) in the quantitative prediction of petroleum potential in both mature and immature basins. Using this distribution it is easy to forecast the quantity and total hydrocarbon resources in any given size interval as well as the forecast of the sizes of undiscovered oil and gas pools and the succession of their discoveries.

The aim of the study is to obtain the respective probability distribution of oil and gas fields using the law of the size distribution of oil and gas pools (truncated Pareto distribution).

Methods: analytical methods of probability theory, statistical test method, statistical processing of empirical data.

Object: petroleum basins, oil and gas reserves of pools and fields, number of pools in each field, laws governing the distribution of the sizes of oil and gas fields.

Results. It was shown that the power-law probability distribution (the classical Pareto distribution, the truncated Pareto distribution) cannot be used as the distribution of sizes of both oil and gas fields and pools. By using statistical testing procedures, the conditional probabilistic distributions of the sizes of oil and gas fields were obtained for a fixed number of pools. Based on the processing of empirical data for four largest petroleum provinces of Russia (Volga-Ural, West Siberian, Timan-Pechora and North Caucasian), we obtained estimates of the distribution of the number of oil and gas pools in each field. The law governing the distribution of the sizes of oil and gas fields was obtained using the law of size distribution of oil and gas pools (truncated Pareto distribution). It was shown that this probability distribution, not being, strictly speaking, a power law, turns out to be very close to it.

\section{Key words:}

Petroleum province, hydrocarbon accumulations, pools, fields, probabilistic distribution of sizes of oil and gas pools and fields, classical Pareto distribution, truncated Pareto distribution.

\section{REFERENCES}

1. Kontorovich A. Eh. Formation of oil and gas in the Earth's crust Petroleum geology handbook. Ed. by N.A. Eremenko. Los Angeles, California, U.S.A., OSI Publications, 1991. pp. 548-569.

2. Kontorovich A.E. Osadochno-migratsionnaya teoriya naftidogeneza: sostoyanie na rubezhe XX i XXI vv., puti dalneyshego razvitiya [Sedimentary-migration theory of naftidogenesis: state at the turn of the $20^{\text {th }}$ and $2^{\text {st }}$ centuries, paths of further development]. Geologiya nefti i gaza, 1998, no. 10, pp. 8-16.

3. Kontorovich A.E., Livshits V.R. New methods of assessment, structure and development of oil and gas resources of mature petroleum provinces (Volga-Ural Province). Geologiya i geofizika, 2017, no. 12, pp. 1835-1852. In Rus.

4. Kontorovich A., Domain V., Livshitc V. Size distribution and dynamics of oil and gas field discoveries in petroleum basins. American Association of Petroleum Geologists Bulletin, 2001, vol. 85 (9), pp. 1609-1622.

5. Kontorovich A.E., Demin V.I. Prognoz kolichestva raspredeleniya po zapasam mestorozhdeniy nefti i gaza [Forecast of the quantity and distribution of oil and gas fields by reserves]. Geologiya i geofizika, 1979, no. 3, pp. 26-46.

6. Kaufman G.M., Balcer Y., Kruit D.A. Probabilistic model of oi and gas discovery. American Association of Petroleum Geologists, 1975, no. 1, pp. 113-142.

7. Shpilman V.I. Kolichestvenny prognoz neftegazonosnosti [A quantitative forecast of oil and gas potential]. Moscow, Nedra Publ., 1982. $215 \mathrm{p}$.

8. Krylov N.A., Aleksin A.G., Baturin Yu.N. Zadachi i put uskoreniya nauchno-tekhnicheskogo progressa pri poiskakh nefti v rayonakh s vysokoy razvedannostyu nedr [Tasks and ways to accelerate scientific and technological progress in searching for oil in mature fields]. Geologiya nefti i gaza, 1986, no. 7, pp. 1-7.

9. Arsiriy Yu.A., Kabyshev B.P., Chuprynin D.I., Shevchenko A.F., Shevyakova Z.P. Prognoz razmerov i chisla neotkrytykh zalezhey UV i metodika ikh poiskov $v$ DDV [Forecast of the size and number of undiscovered hydrocarbon deposits and the method of their exploration]. Geologiya nefti i gaza, 1986, no. 10, pp. 42-46.

10. Schuenemeyer J. H., Drew L.J. A procedure to estimate the parent population of the size of oil and gas fields as revealed by a study of economic truncation. Mathematical Geology, 1983, vol. 15 (1), pp. 145-160.

11. Houghton J.C. Use of the truncated shifted Pareto distribution in assessing size distribution of oil and gas fields. Mathematical Geology, 1988, no. 8, pp. 907-937.

12. Merriam D.F., Drew L.J., Schuenemeyer J.H. Zipf's law: a viable geological paradigm? Natural Resources Research, 2004, no. 13, pp. 265-271.

13. Laherrere J. Distribution of field sizes in a petroleum system: parabolic fractal, lognormal or stretched exponential? Marine and Petroleum Geology, 2000, vol. 17 (4), pp. 539-546.

14. Smith J.L., Ward G.L. Maximum likelihood estimates of the size distribution of North Sea Oil Fields. Mathematical Geology, 1981, vol. 13 (5), pp. 399-413.

15. Burshteyn L.M. Possible control of size distribution of oil and gas fields. Geologiya i geofizika, 2004, vol. 45, no. 7, pp. 815-825. In Rus.

16. Livshits V.R. Imitational stochastic model of lateral migration of hydrocarbons. Geologiva i geofizika, 2014, no. 5, pp. 906-917. In Rus.

17. Livshits V.R. Lateral migration of hydrocarbons as a possible mechanism for the formation of the power distribution of their clusters by mass. Geologiya i geofizika, 2017, no. 3-4, pp. 372-383. In Rus.

18. Rodkin M.V., Rundkvist D.V. Geoflyuidodinamika. Prilozhenie $k$ seysmologii, tektonike, protsessam rudo $i$ neftegeneza [Geofluidodynamics. Application to seismology, tectonics, ore processes and oil and gas]. Dolgoprudny, Intellekt Publ., 2017. 282 p.

19. Demenok S.L. Fraktal: mezhdu mifom i remeslom [Fractal: between myth and craft]. St-Petersburg, Strata Publ., 2018. 87 p.

20. Feller V. Vvedenie $v$ teoriyu veroyatnostey $i$ ee prilezheniya [Introduction to probability theory and its applications]. Moscow, Mir Publ., 1984. Vol. 2,751 p.

Received: 22 February 2020.

\section{Information about the authors}

Valery R. Livshits, Dr. Sc., chief researcher, Trofimuk Institute of Petroleum Geology and Geophysics, Siberian Branch Russian Academy of Sciences. 